\title{
Editorial Sexual dysfunction and its relation to heart disease
}

\author{
International Journal of Impotence Research (2005) 17, S1-S3. doi:10.1038/sj.ijir.3901421
}

What do cardiologists have to do with erectile dysfunction? Even though this seems far from our daily clinical routine, a closer look and interrogation among our own patients revealed that the majority of patients with underlying chronic cardiovascular disorders are suffering from erectile dysfunction. The close association of coronary artery disease and vasculogenic erectile dysfunction is a well-known phenomenon, however, it represents an example where scientific evidence did not translate into clinical practice and routine. How many cardiologists do really ask their patients about sexual problems? In addition, how many patients with ED and concomitant cardiac problems are not treated for ED because they are considered as high risk for sexual activity as well as for using drugs such as PDE-5 inhibitors. Even though the pathophysiologic mechanisms for ED are well known among urologists dealing on a daily base with ED patients, the majority of cardiologists do neither spent enough time to read about ED nor to treat ED, lacking awareness how significant sexual dysfunction impacts our patients' lives, sometimes even more than the experience of a myocardial infarction or any other cardiovascular (even life-threatening) event. Patients might not tell us cardiologists about this particular problem, and we are not asking about it.

Common knowledge is that sexual problems increase with age as well as with the development of illnesses and particular chronic diseases such as diabetes, kidney failure and, most notably, heart diseases. Despite well-known data from the historic Alfred C Kinsey report from 1948 which gained recently much more attention due to the release of the movie Kinsey-lets talk about sex (as well as the Starr report from 1981) revealing that people above $65 \mathrm{y}$ of age were on average once per week sexual active (and women between the ages 61 and $91 \mathrm{y}$ had on average 1.4 times per week) sexual activity decreases in frequency with age. In particular in view of the aging baby boomer generation currently in their mid-forties there will be an overwhelming number of people suffering from sexual dysfunction in the coming years, which is associated with depression and reduced quality of life. On the other hand, sexual performance problems are still a taboo: several people never talk to their physicians about these problems. Even if, unfortunately many physicians lack adequate education and knowledge how to address sexual dysfunction, especially in older patients and patients with concomitant cardiovascular diseases. Sexual dysfunction is caused by different conditions, with even more differences between men and women. In males, sexual dysfunction normally refers to erectile dysfunction, which is the inability to gain or maintain penile erection with resulting inability to successfully perform coitus. Premature ejaculation or anorgasmy and reduced desire can be different presentations of sexual dysfunction. In women, sexual dysfunction more often might represent lack of vaginal lubrication, anorgasmy, and painful intercourse or reduced arousal caused by lack of libido. Interestingly, little is known about sexual dysfunction in women and just recently some researchers have addressed this problem more intensively. In general, there are several distinct reasons for sexual dysfunction, in particular in men. These are vascular, endocrine, hormonal, psychogenic/psychosocial and/or neurogenic causes and potential drug side effects, in addition to rare traumatic cases. It is estimated that sexual dysfunction is caused by vascular conditions in more than $80 \%$ of all cases, that is by inadequate blood supply and vascular dysfunction. Since there is a $52 \%$ probability for sexual dysfunction in men between the ages of 40 and $70 \mathrm{y}$ (ie, every second individual might develop sexual dysfunction), these numbers likely are going to increase due to aging and prolonged survival even with critical heart conditions. Therefore, sexual dysfunction represents a major medical as well as economic and psychosocial problem. In particular in the elderly and in patients with cardiac diseases, there are several problems for adequate treatment of sexual dysfunction, which are summarized as follows:

1. Fear: Especially following a cardiac event such as a heart attack or symptoms of chest pain or after a procedure such as a cardiac catheterization or bypass surgery, many people are afraid of resuming sexual activity due to a fear of a heart attack. In fact, the real-world incidence of having a heart attack or life-threatening arrhythmia during sexual activity is relatively low: due to a report from $1996<1 \%$ of all heart attacks happen during sex, and sudden death during sex occurs in only 1$3.3 \%$ of all cases of sudden death.

2. Concomitant medication: Several drugs have potential side effects such as the development of 
$\mathrm{S} 2$

erectile dysfunction or decreased libido. It is always worth the effort to evaluate potential drug side effects and exchange the class of drugs, if possible, to others with fewer side effects on sexual function.

3. Risk factors: The risk factors known to be associated with the occurrence of atherosclerosis, myocardial infarction and stroke are identical to the risk factors associated with occurrence of erectile dysfunction, such as diabetes, smoking, high cholesterol and high blood pressure. Adequate control of risks is essential in prevention of potential life-threatening conditions but also in improving sexual dysfunction.

4. Performance capability: To perform sexual intercourse, heart rate and blood pressure rises, on the average for $5-15 \mathrm{~min}$ and the overall hemodynamic challenge for the heart requires only $3-5 \mathrm{ml} / \mathrm{min}$ of the total cardiac output for male erection. The average sexual activity level equals climbing two flights of stairs. Most patients with preserved left ventricular function after myocardial infarction can resume sexual activity. Interestingly, $25 \%$ of all men cease all sexual activity and $50 \%$ report decreased interest. Even after successful balloon angioplasty of bypass surgery for coronary artery disease, $36 \%$ of men reported decreased sexual activity. In men with heart failure, $60 \%$ seem to be no more interested in sex and $40 \%$ believe that they should not be sexually active in order to prevent potential damage to their heart.

5. Medication interaction: Since introduction of the PDE-5 inhibitors Viagra ${ }^{\mathbb{B}}$, Levitra ${ }^{\circledR}$ and Cialis ${ }^{\circledR}$ and officially published statements of celebrities such as Senator Bob Dole or Playboy founder and editor Hugh Heffner regarding their use and success with these drugs to improve sexual performance and maintain an active sex life, many reports described the potential life-threatening actions of these drugs in patients with heart problems. By reviewing FDA reports and publications in details, it becomes obvious that the absolute contraindication for all PDE-5 inhibitors is the concurrent use with NO donating agents such as nitrates. Nitrates are used as oral tablets, sublingual tablets or spray or capsules or as transcutaneous patches to treat chest pain/ angina pectoris. If nitrates and PDE- 5 inhibitors are taken together, the combination of the two can result in a cumulative drop in blood pressure that might lead to death in patients with coronary artery disease. Patients with known coronary artery disease, who are in a stable condition and not using nitrates, can use PDE-5 inhibitors.

Owing to advances in understanding the pathophysiologic mechanisms in particular with regard to its vascular causes and pharmacologic advances, most cases of sexual dysfunction can be treated.
The University of Texas Medical Branch in Galveston, Texas, organized the very first conference on the association between sexual dysfunction and heart diseases, entitled 'Sex and the Heart', which was held April 23, 2005 at the Galveston Convention Center at the Texas gulf coast. An internationally recognized faculty of physicians and researchers were gathered to exchange recent scientific knowledge how to address sexual dysfunction in patients with cardiac problems, which currently and most likely even more in the near future represents the majority of patients with sexual dysfunction. Topics such as sexual dysfunction as an early marker for coronary artery disease, sexual function and dysfunction in the elderly, future treatment options such as gene and stem cell therapy and a more detailed understanding of sexual dysfunction in women were presented and discussed.

It is the aim of this supplement of the International Journal of Impotence Research to present the variety of topics of this meeting, important for cardiologists, internists and primary care physicians not that familiar with the problems of ED and urologists not that familiar with the cardiovascular problems of most of their current and future patients on how to deal with patients with chronic cardiovascular conditions and erectile dysfunction. This supplement represents the first one on the close association between sexual dysfunction and cardiovascular disorders. The articles represent a variety of different aspects of the topic, from basic research data to clinical routine scenarios of patients with sexual dysfunction. In the current supplement Dr Billups presents data form his group which was among the first demonstrating evidence that erectile dysfunction is associated with endothelial dysfunction and that ED therefore can be an early sign and marker for coronary artery disease; Dr Kloner created a hypothetical patient and explores the evaluation and treatment options in different case scenarios as recommended by the II. Princeton Consensus Panel; Dr Camacho, a clinical geriatrist, gives an overview about sexual problems with increasing age; Dr Kadowitz and his group summarizes their exciting experimental data mainly in rat models using gene therapy and stem cell therapy as potential treatment options for ED; Dr Berman gives a broad overview about female physiology required for sexual function and the pathophysiologic concepts behind sexual dysfunction in women; Dr Jones lifts us up to space and presents interesting data about genitourinary and reproductive functional changes during the NASA space flight missions. Finally, our group summarizes the close connection between sex and the heart from the cardiologist's view as well as a brief presentation of our multidisciplinary approach to high-risk cardiovascular patients with erectile dysfunction. A critical review of patients with heart failure, who are always considered as high risk for sexual 
dysfunction as well as for any treatment modulates, is included.

This supplement of the International Journal of Impotence Research is intended to give the reader a broad overview about the close association between sexual dysfunction and underlying cardiovascular disorders, new assessment and treatment approaches, future (research) options and open questions for these increasing problems in our society.
Moreover, we hope to create more interest among health care professionals for this important field to gather more attention for our annual meetings and future publications on 'Sex and the Heart'.

ER Schwarz

Division of Cardiology, Department of Internal Medicine, The University of Texas Medical Branch, Galveston, TX, USA 